\title{
Irritierende Artefakte: Wie sich die handlungsbezogene Virtualität in Modellen zeigt
}

Insbesondere in der sich seit etwa 2012 vollziehenden zweiten Welle der Diskussion und Entwicklung von virtueller Realität (die erste vollzog sich etwa Mitte der 1990er Jahre) werden diese immersiven Techniken als Instanzen gewürdigt, die es erlauben, sich von der Außenwelt immer besser abzukapseln. Ich folge französischen Denkern darin, dass das Virtuelle jenseits des rein Technischen steht und keinen Gegensatz zum Realen bildet. Mit Blick auf Modelle, und hier insbesondere auf Architekturmodelle, binde ich die Virtualität nicht an die Optik, wie es die Filmwissenschaftlerin Anne Friedberg unter Herleitung historischer Traditionen einleuchtend unternimmt, und auch nicht an eine (technologische) Medienspezifik (Friedberg 2006, 8-9). Vielmehr versuche ich, das Virtuelle als etwas (Daseinsweise in Modellen) vorzustellen, das implizit Handlungsangebote zu unterbreiten vermag. Damit rücke ich das Virtuelle in die Nähe der Affordanzen, dem Praxigenen. Dieses aktionsbezogene Virtuelle der Artefakte erfährt im Umgang eine Aktualisierung. Dabei kann der Fall eintreten, dass der Umgang nicht zum Modelltypus passt. Es kann fernerhin dazu kommen, dass die modellhaften Artefakte dergestalt ausfallen, dass sich das Handlungsangebot daran so stark verschiebt, dass man hierfür erst einmal eine neue Funktionalität finden müsste. Darum veranlassen die im Folgenden vorgestellten architektonischen und künstlerischen Beispiele von Peter Eisenman, Mario Botta, Pierre Huyghe und Florian Dombois zunächst zum Schmunzeln und oder zum Ärgern. Materielle Umsetzungen von architektonischen Konfigurationen, die zuvor auf Papier oder im Computer vorlagen, sind an sich wahrlich nichts Ungewöhnliches - warum stechen diese heraus? Sie dienen hier dazu, Darstellungskonventionen als handlungsbezogen perspektiviert zu konnotieren. Wenn dem so ist, lohnt es auch bei digitalen 3D-Rekonstruktionen - die vermeintlich verzerrungsfrei abbilden - darauf zu achten, wie sich die Handlungsperspektivierung als virtuelle Instanz auswirkt.

\section{Provozierende Modellierungen}

Zunächst stelle ich die vier architektonischen bzw. künstlerischen Beispiele kurz vor, um danach drei Argumentationslinien zu verfolgen, die in eine 
ähnliche Richtung weisen und eine Diskussion digitaler Rekonstruktion vorbereiten.

\subsection{Gebaute Axonometrie - Peter Eisenman}

Einen prominenten Beitrag zum Nachdenken über die Projektion in 3D-Gebilden leistet der US-amerikanische Architekt Peter Eisenman im Rahmen seines entworfenen, aber letztlich nicht gebauten Projekts House X (1975-1978). Abbildung 1 zeigt eine axonometrische Projektionsdarstellung des Gebäudes auf Papier. Die Axonometrie ist eine im Ingenieurwesen schon länger, in der Architektur insbesondere seit den 1920er Jahren beliebte und übliche Darstellungsweise für Entwurfsgegenstände. Es handelt sich um ein Verfahren der darstellenden Geometrie, Körper in einer Zeichenebene wiederzugeben. Axonometrische Diagramme sind sachlich insofern, als es sich um eine distanzierende Projektionsart handelt, bei der Betrachter_innen nie im Bild stehen (Schneider 1981). Auch bei Eisenmans dreidimensionalen axonometrischem Konstrukt (Abb. 2) ist folglich kein Blickpunkt in Bezug auf die Architektur gegeben (denn es wird weniger Raum dargestellt als Maßverhältnisse unverzerrt ablesbar gemacht), sondern nur in Bezug auf die Darstellung der Architektur. Eisenman transferiert diese Projektionsart in den lebensweltlichen dreidimensionalen Raum. ${ }^{1}$ Zweifellos wird ein Blatt mit einer Zeichnung auch in die Hand genommen, gedreht und seitlich betrachtet. Dadurch bekommt man aber keine zusätzlichen Details offenbart, die bei einem frontalen Zublick nicht auch prinzipiell schon vorhanden wären. Das Objekt des House $X$ zeigt sich von einem bestimmten Blickwinkel aus der Zeichnung entsprechend (Abb. 2), aber von allen anderen Seiten (Abb. 3) präsentiert es notwendigerweise zusätzliche Informationen zum Bau. Damit ergibt sich eine Spannung zur angewandten zeichnerischen Projektionslogik, die gerade Räumlichkeit suggerieren bzw. zwischen 2D und 3D vermitteln sollte und - in den Realraum transferiert - ihre Aufgabe verliert und eine neue Bestimmung erhalten müsste. Über eine mathematische Transformation kippte Eisenman alle Vertikalen um 45 Grad in dieselbe Richtung. Damit tritt es auch in ein Spannungsverhältnis mit dem angedachten Bau, den es - einer Anamorphose nicht unähnlich - nur unter einem einzigen Blickwinkel normgerecht zu repräsentieren vermag. Bei der Papiervariante der Axonometrie ist dieser Blick-

1 Es sei erwähnt, dass der Informatiker Gershon Elber vom Technion in Haifa eine Herausforderung darin fand, die Zeichnungen Eschers mit Hilfe von 3D-Druckern als dreidimensionale Konstrukte zu realisieren. Vgl. Elber undat. 


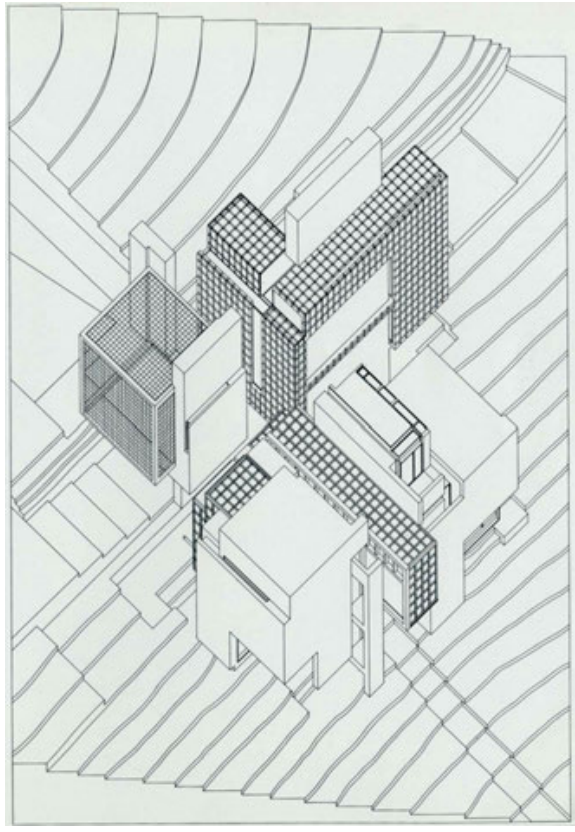

Abb. 1: Peter Eisenman: House $X$, 1975-1978. Axonometrische Zeichnung des Schemas H. Quelle: Eisenman 1983, 159, Abb. 209.

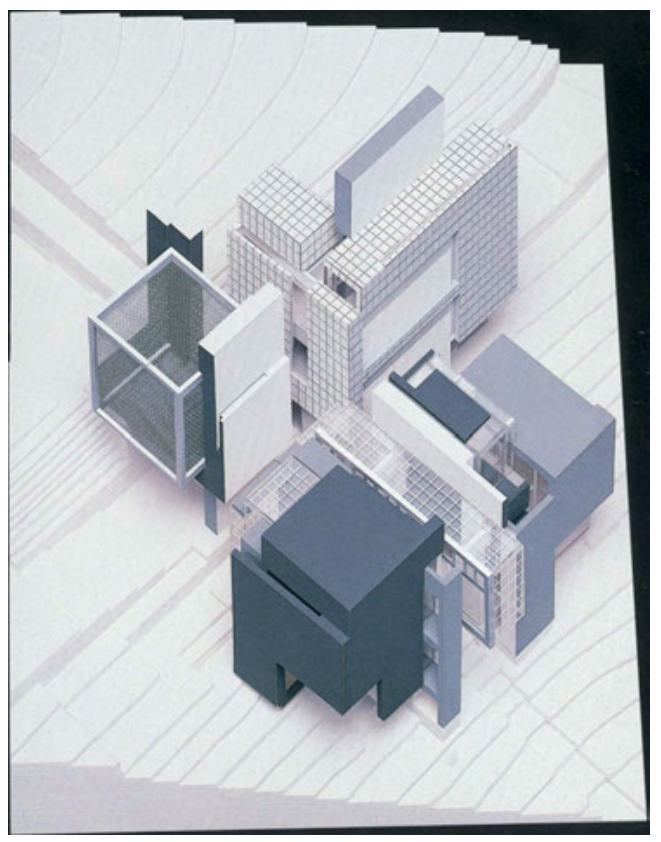

Abb. 2: Peter Eisenman: House $X$, 1975-1978. Axonometrisches Modell des Schemas H. Ansicht von Nordost. Quelle: Eisenman 1983, 163, Abb. 211. 


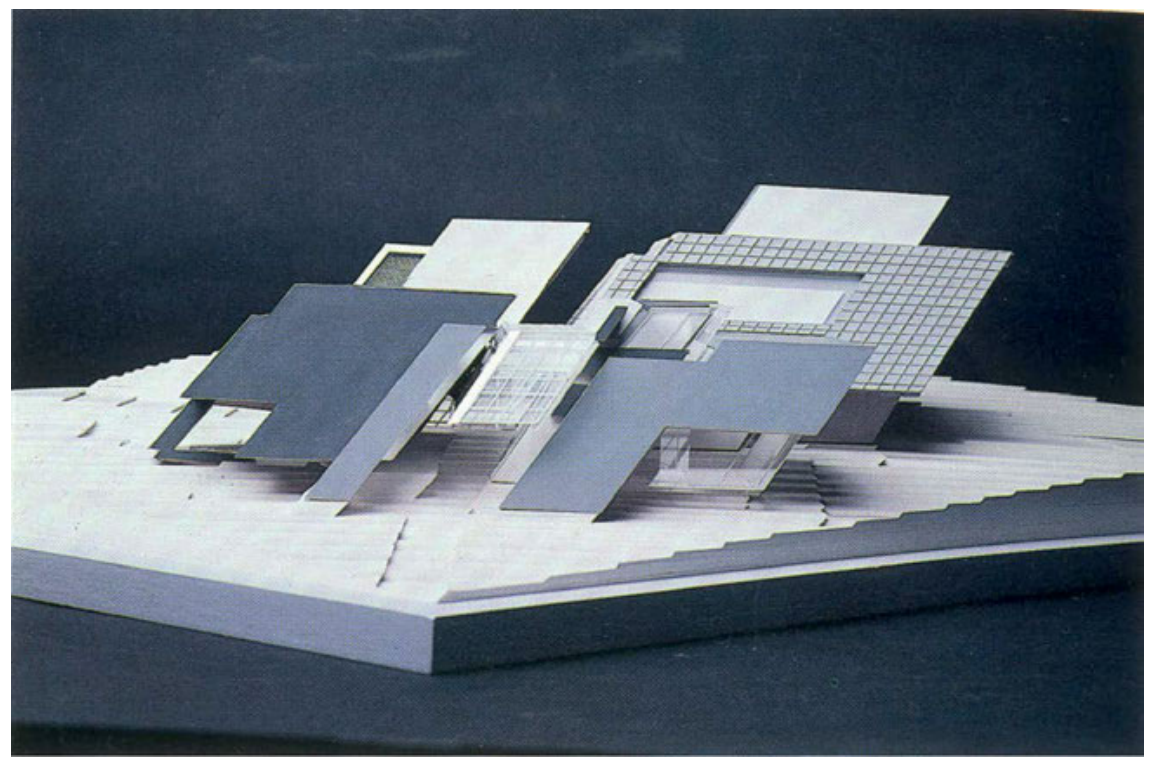

Abb. 3: Peter Eisenman: House X, 1975-1978. Axonometrisches Modell des Schemas H. Ansicht von Norden. Quelle: Eisenman 1983, 164, Abb. 213.

winkel bereits ,eingebaut', beim axonometrischen dreidimensionalen Objekt auch, aber als Objekt im Raum erlaubt es zudem allseitige Zublicke. Hier werden ein Einsehen und ein Eingriff eher vereitelt, man hat die Entzerrung nur, wenn man sich auf einen Blickpunkt diszipliniert, der in der Zeichnung schon inkorporiert wäre. Damit wäre der Vorzug der dreidimensionalen Darstellung karikiert. Ein wenig begeisterter Kritiker - der den Blick aus der Vogelperspektive verinnerlicht hat - schrieb dazu: „Looking down to this helpless model, which resembles a crab squashed on a beach, one can only admire Eisenman's success at this task [of leaving architecture with no task but to symbolize impotence]“ (Pommer 1981, 10). Es wäre auch möglich zu sagen, dass sich für die Betrachter_innen die Frage stellt, ob es sich um ein schiefes Modell eines orthogonalen Entwurfs oder um ein ,normales‘ Modell eines schiefen Hauses handelt. Die inhärente Ambiguität in axonometrischen Zeichnungen nahm sich Eisenman im Projekt El Even Odd (1980) explizit vor. Ein Würfel, dem ein würfelförmiges Achtel fehlt, dient ihm als ,L'-förmiger Baukörper, den er in vier Varianten auf Weisen verzerrt, dass er axonometrisch immer noch exakt gleich repräsentiert werden kann. Bei geeigneter Wahl der Projektionsrichtung, so der Architekt Daniel Lordick, kann man der Axonometrie nicht ansehen, ob das Objekt gerade (,even') oder schief (,odd') 
ist (Lordick 2005, 142-143). Eisenmans Modell von House X erntete Kritik, denn es entspricht als Gesamtkonfiguration keiner Norm und funktioniert nur als einseitig betrachtetes, seine ,konzeptuelle Essenz' ist die Zeichnung. Oder es thematisiert die Kontingenz der Normierung. Mit ihm legt er indirekt nahe, dass auch 3D-Gebilde Projektionslogiken beinhalten, d. h., dass sie nicht per se ,verzerrungsfrei‘ sind. Eisenmans Objekt zählt heute zu den berühmtesten Architekturmodellen des zwanzigsten Jahrhunderts.

\subsection{Gebauter Aufriss - Mario Botta}

Das nächste Beispiel stammt vom Schweizer Architekten Mario Botta. Sein S. Carlo alle Quattro Fontane (Abb. 4) bezieht sich auf einen historischen Bau, nämlich auf die 1638-1641 erbaute gleichnamige Kirche des Barockarchitekten Francesco Borromini (1599-1667). Zu dessen 400. Geburtstag erstellte Botta in Kollaboration mit der Università della Svizzera Italiana im Jahre 1999 ein - ja was? Das Ringen um Kategorien ist den Kommentaren deutlich zu entnehmen. In seiner eher literarischen Besprechung nennt der Architekt Emanuele Saurwein das Monument einen „Schauspieler“, ein „geklontes Phantom“, ein „Spektrum“ und „Gespenst“, ein „Scheinbild“ oder „Schatten eines Schattens“ (Saurwein 2001). Auch der Philosoph Nicola Emery findet eine ganze Reihe von Begrifflichkeiten dafür: „Bild“, „großes Simulakrum“, „traumähnliches und enigmatisches Ornament“ oder „Repräsentation-Modell-Kunstgriff“ (Emery 1999). Für den Kunsthistoriker Rolando Bellini ist es ein „Artefakt in Lebensgröße“, ein „exzentrischer Replikant“ und ein „gigantischer hölzerner Querschnitt“ (Bellini 2000, 15, 33 und 41). Laut dem Architekten selbst sei das Gebäude wie eine szenografische Konstruktion (Botta 1999, 14), oder ein „non-finito“ bzw. „Fragment“. ${ }^{2}$ Andernorts spricht man von einer Darstellung einer Sektion aus Holz, die sich wie eine „Architektur und Szenografie, wie eine Skulptur und Installation“ darbiete (Sala und Cappellato 2003, 151).

Was wurde 1999 am See von Lugano eigentlich gebaut? Man errichtete einen in Holz ausgeführten Querschnitt durch die Schmalseite der römischen Kirche in Originalgröße des Gotteshauses und mit einer für Modelle nicht unty-

2 Die Rede vom Fragment erstaunt, denn der Schnitt passierte im Computermodell, er ist in der Ausführung aber ein additiv Gebauter. Damit markiert diese Vorgehensweise den Gegenpol etwa zum splitting des Künstlers Gordon Matta-Clark, der mit seinen building cuts bestehende Bausubstanz zerschneidet. 


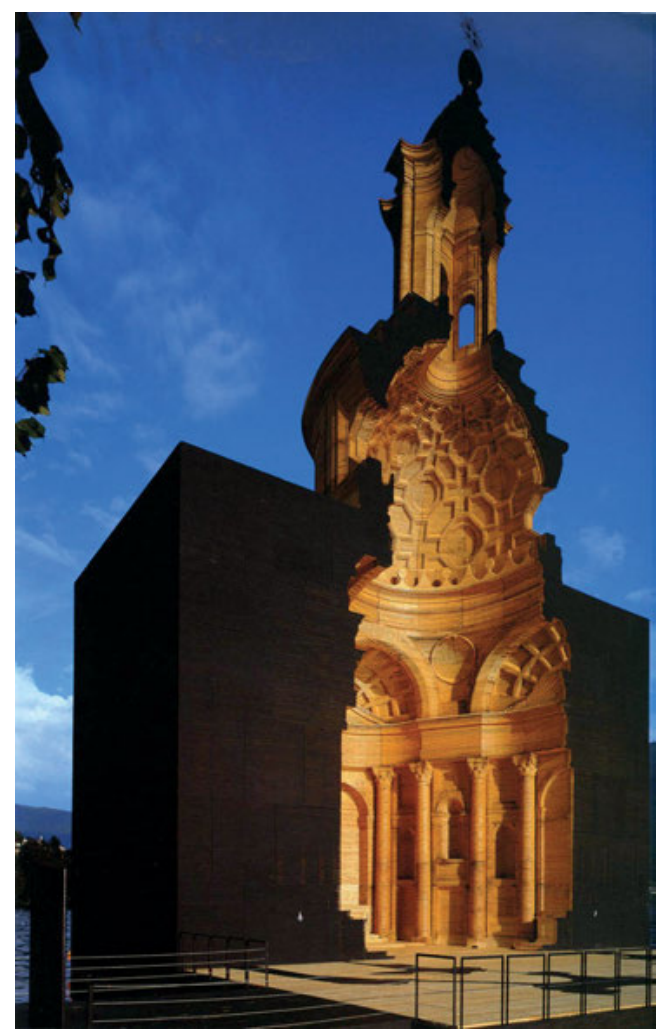

Abb. 4: Mario Botta: S. Carlo alle Quattro Fontane, 1999. Quelle: Cappellato 1999, 241.

pischen Schichtbauweise - man könnte auch sagen: einer gröberen horizontalen Auflösung (Von Moos 1999, 36).

Obwohl es im ersten Moment so aussieht, als würde Botta einen historischen Aufriss bauen, entnahm er die Rauminformationen den neuesten Datenerhebungstechniken. Unter der Leitung von Alessandro Sartor von der Università la Sapienza in Rom wurde 1998 über die Fernerkundungstechnik der Fotogrammetrie (Bildmessung) eine virtuelle dreidimensionale Rekonstruktion von S. Carlino erstellt und daraus ein Holzmodell im Maßstab 1:33 produziert. Letzteres wiederum bildete den Ausgangspunkt für Bottas Bau, den der Architekt selber auch als ,Holzmodell' bezeichnet und der mit seinen über 33 Metern Höhe und je 22 Metern Breite und Tiefe die Originalgröße aufwies. Für die einzelnen 4,5 cm starken zu profilierenden Holzplanken fertigte man über 36.000 Umrisszeichnungen an und druckte sie auf insgesamt $15 \mathrm{~km}$ Papier aus (Saurwein 2001, 22-23). Zuvor 
jedoch wurde ein kleineres Modell in der Technik des Lamined Object Manifacturing (LOM) zu Testzwecken gefertigt (Vgl. Bellini 2000, 19). ${ }^{3}$

Als Tribut an die urbane Lage der in eine Häuserfront integrierten Barockkirche sieht man die Ausblendung der angrenzenden Gebäude als homogenen dunklen Block, quasi als ,unbestimmt' markiert. Dieser Kubus ist bis zur Höhe des Kuppelansatzes hochgezogen. Da die Dachbekrönung auch im Original freisteht und damit Information zur außenseitigen Gestaltung gegeben ist, wurde auch die Holz(re)konstruktion ab der Kuppel innen und außen ausgeführt. Dies entspricht dem Modus des ,einsamen Modells‘. Zwar ist es hier im Realraum integriert, aber dennoch in den See hinausgesetzt und damit von allem anderen abgesetzt.

Als Tribut zur Aufrisszeichnung, bei der häufig die Mauerstärke schraffiert oder eingedunkelt repräsentiert ist, erfolgt an der Schnittstelle die Einschwärzung des ansonsten naturbelassenen Holzes. Auf dem Boden der Plattform vor dem Holzbau ist ebenso in Schwarz die fehlende zweite Hälfte des Baus als Grundriss angedeutet.

Anders als in den meisten reproduzierten Schnitten entschloss sich Botta, das Gebäude mittig über die kurze Achse zu teilen, obwohl die Längsachse mehr Informationen zur römischen Kirche offenbart hätte. Dazu schreibt Carlo Bertelli:

Un taglio sulla lunghezza avrebbe dato sicuramente maggiori informazioni, che è il motivo per cui furono eseguiti i rilievi settecenteschi, ma qui lo scopo era un altro. La metà dell'interno della chiesa di legno si vede ora da molto lontano, e così la conca dell'abside si apre verso uno spazio infinito. Di conseguenza la profondità della chiesa aumenta ben al di là della costruzione presa a modello.

(Bertelli 1999, 23)

Ein großes Modell könnte viele Details haben, aber in Bottas Modellbau ist dies nicht der Fall, ohnehin wurde es eher auf Fernwirkung hin konzipiert. „A Lugano una cosa [sezione di un edificio] vista su un foglio stampato è diventata una realtà per metà uguale a quella vera - una follia“ (Panza di Biumo 1999). Die Verrücktheit bestand im Verrücken von Darstellungskonventionen. Dies führte zu Empörung, Verstörung oder zur Anerkennung einer unterstellten Subversion. Bottas Arbeit als ,skulptural‘ oder ,installativ‘ zu bezeichnen, bedeutet, es tendenziell aus der Domäne des architektonisch Modellhaften - des Angewandten - hinauszukomplimentieren und in den Bereich der freien bildenden

3 Dabei handelt es sich um ein Verfahren des rapid prototyping, bei der das Objekt schichtweise aus Papier aufgebaut wird, indem man die einzelnen Scheiben der Kontur nach ausschneidet. 
Künsten zu verschieben. ${ }^{4}$ Die hier vorgeführten Verbindungen von Bildlichem und Modellhaftem folgt den Konventionen nicht hinreichend. Ein Aspekt war mit der Fernwirkung angesprochen und dieser beinhaltet teils schon den zweiten, nämlich, dass sich das Konstrukt qua seiner aus der Nähe unübersichtlichen Größe dem umgestalterischen Zugriff entzieht. Manche Eigenschaften des Hilfskonstrukts des Aufrisses bzw. Querschnitts, der die Geometrie der Fläche, das Maß anstatt des Raums darlegt, wurden effektiv räumlich gebaut. Da sich Botta bei der Größe am effektiven Vorgängerbau und nicht an papierenen Orthogonalplänen orientierte, wird dies für eine Inspektion der Maße unübersichtlich. Hier kann man nicht einfach den Kopf bewegen und alles von allen Winkeln her besehen, wie etwa bei einem handlichen Konstrukt. Kleinere dreidimensionale Modelle vermitteln noch die Idee, dass die Darstellung ,angeschnitten' ist, um den Blick auf das Innere freizugeben, um eine Einsicht zu gewähren. Wenn dies aber in Originalgröße gebaut ist, fühlt es sich nicht mehr als kognitiv-heuristischer Schachzug in der bildlichen Darstellung an, sondern als ein versehrtes Gebäude, das an einer Seite keinen Abschluss besitzt, wobei aber weniger die Offenheit der Konche frappiert als die surreale Präzision des vertikalen Schnittes ungeachtet aller Diversität der baulichen Elemente. So kommen der Standpunkt der Betrachter_innen und notgedrungen deren Bodenverhaftetheit stärker zum Tragen. An die Stelle einer Übersicht tritt die Beeindruckung.

\subsection{Aufgeführtes Kantenmodell - Pierre Huyghe}

Das dritte Beispiel stammt vom französischen Künstler Pierre Huyghe. Er bedient sich in This is not a time for dreaming (2004) eines - für die Thematisierung eines Schaffensprozesses - ungewöhnlichen Formats: eines Marionettentheaterspiels. Um genau zu sein, ist es das Format des filmisch aufgezeichneten Puppenspiels. Huyghe greift das Carpenter Center von Le Corbusier im Harvard Campus auf, indem er die Entstehungsgeschichte entlang einer ganzen Typologie von Entwurfsartefakten auffädelt. Dadurch, dass Huyghe an die Abfolge von Modellen auch jeweils Handlungen knüpft, suggeriert er dessen Darlegung eines Entwurfszusammenhangs. Das Theater - selbst eine Modellsituation - begünstigt eine Kondensierung der Herstellungsprozesse in Form von bildlichen Artefakten als Symbole für Entwurfsstadien. Diese eingängigen visuellen Pointen erklären sich möglicherweise daraus, dass für alle Elemente im Bühnenstück galt, dass sie als

4 In diesem Zusammenhang wird öfters das Nur-sich-selbst-Zeigen des Holzbaus hervorgehoben, vgl. z. B. Minazzi 2000, 53 und 59. 
filmisches Motiv taugen mussten. In Huyghes Film zeigen sich die architektonischen Artefakte als frei flottierend, sich unentwegt transformierend, sich errichtend (vgl. Hinterwaldner 2017). Sie mussten nicht nur fotogen, sondern bewegtbild-adäquat sein. Für die zeitbasierte Inszenierung wurden die gezeigten Modelle extra modular gefertigt. In Abb. 5 sehen wir ein Kanten- oder Drahtgittermodell neben dem träumenden Le Corbusier. Es erscheint zunächst wie eine leichte Zeichnung am Boden, die sich aber erstaunlicherweise aufrichtet, die obersten Stockwerke zuerst entfaltend. Die architektonischen Teile geben sich nacheinander zu erkennen. Um diesen Effekt zu erzielen, legte Huyghe Wert darauf, das schwarze Gestänge aus unterschiedlichen Teilen fertigen zu lassen. Dies alles schilderte er den Verantwortlichen des Requisitenbaus detailreich am Telefon. Durch diese Maßnahme konnte man die Teile auch unabhängig voneinander zu unterschiedlichen Zeiten hochziehen. Huyghe überführt dieses Konstrukt über die vierte Dimension der Zeit von einer Art Plan (2D) in ein Kantenmodell (3D). Die Genese der Architektur(skizze) als Entfaltungsprozess zu präsentieren, als ein In-die-Höhe-Wachsen, aber die oberen Stockwerke allen anderen voran ist weit realitätsferner als es im Film erscheint. Die Projektion eines Kantenmodells ist nämlich keine Faltung eines gebauten Drahtgestänges. Beim Kollabieren auf zwei Dimensionen passiert nicht, wofür die flächige diagrammatische Darstellung prädestiniert wäre, nämlich die vertikale Dimension wegzulassen. Bringt man das Kantenmodell über eine Projektion in zwei Raumdimensionen, ergäbe sich nie

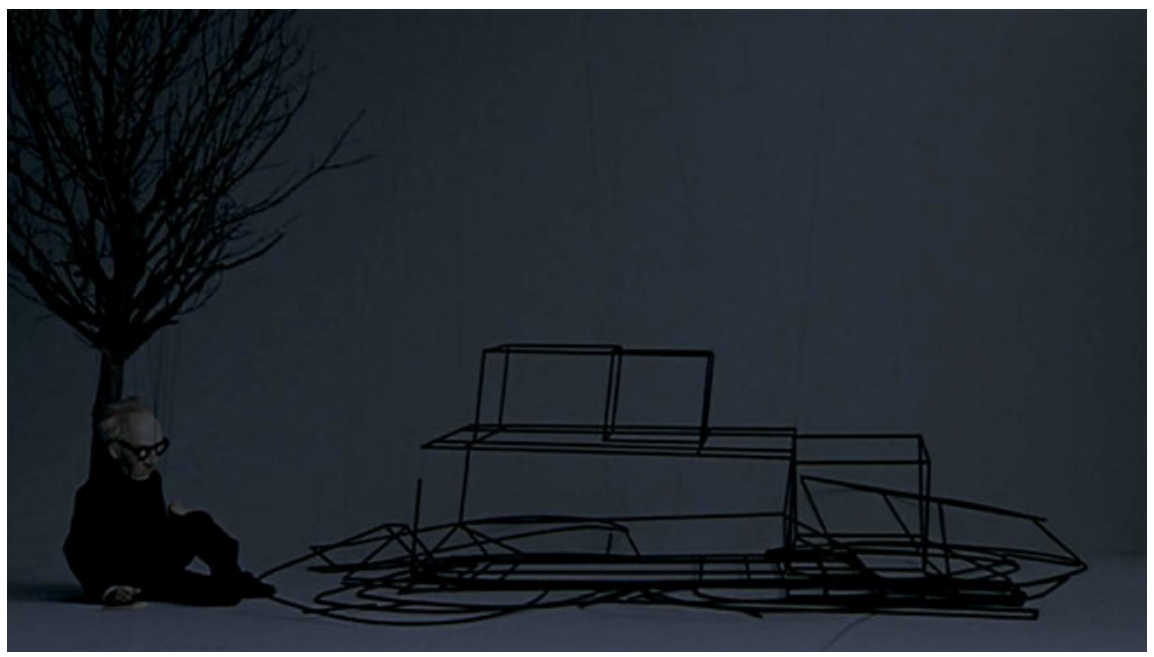

Abb. 5: Pierre Huyghe: This is not a time for dreaming, 2004. Still vom Kapitel IV: Le Corbusier träumt ein Drahtgittermodell. Mit freundlicher Genehmigung des Künstlers. 
diese Ansicht auf dem Boden in Huyghes Bühnenspiel, weil sich die Längen der Stäbe des materiellen Gestänges nicht verkürzen, sondern in voller Länge liegen bleiben, aber dank der beweglich konstruierten Gelenke die Lage zueinander ändern können, sodass die Ansammlung auch (relativ) flach am Boden liegen kann.

\subsection{Gebaute Billboards - Florian Dombois}

Das vierte und letzte Exempel geht auf den deutschen Künstler Florian Dombois zurück. Er realisierte ein „Kunst am Bau“-Projekt am 2010-2014 rekonstruierten Stadtschloss Potsdam, in das der Landtag Brandenburg einzog. Für den Herrenhof der Anlage konzipierte Dombois zwei Pavillons, die auf die Rasenflächen gesetzt wurden. Der Titel der Arbeit, Zugabe (2014), spiegelt auf mehrfache Weise die Logik des Rekonstruktionsbaus, bei der der Architekt Peter Kulka die Außenmaße des alten preußischen Stadtschlosses (1662-1674 im Stile des Frühbarocks erbaut und 1744-1752 von Georg Wenzeslaus von Knobelsdorff in den Rokoko überführt) beibehielt, dessen historische Außenfassaden imitierte sowie erhalten gebliebene Originalsubstanz integrierte, aber eine spätmoderne Innenraumgestaltung dahinter setzte. Auch hatte das Gebäude gewachsenen Raumanforderungen zu genügen, die es nötig machten, dass der Innenhof etwas verkleinert wurde. „Hier werden die Fassadenflächen gestaucht. Dabei entsteht im Innenhof eine Art Modellsituation. Das Haus wird zum Architekturmodell seiner selbst“ (Dombois 2012, 1). Für Dombois stellte dies ein Faszinosum dar und es kam ihm als Ausgangspunkt entgegen, mit der Kulissenarchitektur als ,Material` zu arbeiten. Die Pavillons (Abb. 6) wurden nicht räumlich-vollplastisch gebaut, sondern bestehen aus bemalten Aluminiumflächen, die im rechten Winkel zueinander gestellt wurden. Als Motiv zeigen die Pavillons die fotografische Reproduktion des Zentralovals der Tafelrunde von Sanssouci. Somit gelang es dem Künstler, der Winterresidenz von Friedrich II. mit seiner Zugabe eine Verlängerung zu dessen nahe gelegener prachtvollen Sommerresidenz Sanssouci (1745-1747) herzustellen, den derselbe Architekt Knobelsdorff verantwortete. Die Künstlerin Annette Paul würdigt Dombois' Kühnheit, „einen fast heiligen Raum zu zerschneiden, das Oval im Marmorsaal, das Herzstück von Friedrichs Tafelrunde. Und das mit einem Kreuz. Das ist schon gewagt“ (Jäger 2012, unpag.). Professionelle Aufnahmen des Ovals von außen, aus leichter Untersicht mittig von der zentralen Treppe aus menschlicher Augenhöhe, wurden in eine hochauflösende Datei (mit $20.000 \times 20.000$ Pixeln) zusammengeführt (Dombois 2017). Zwei Theatermaler sollten nun alle insgesamt acht Flächen der Pavillons mit diesem einen Motiv bemalen. Durch die unterschiedlichen Größen der Pavillons, die Spiegelung in 


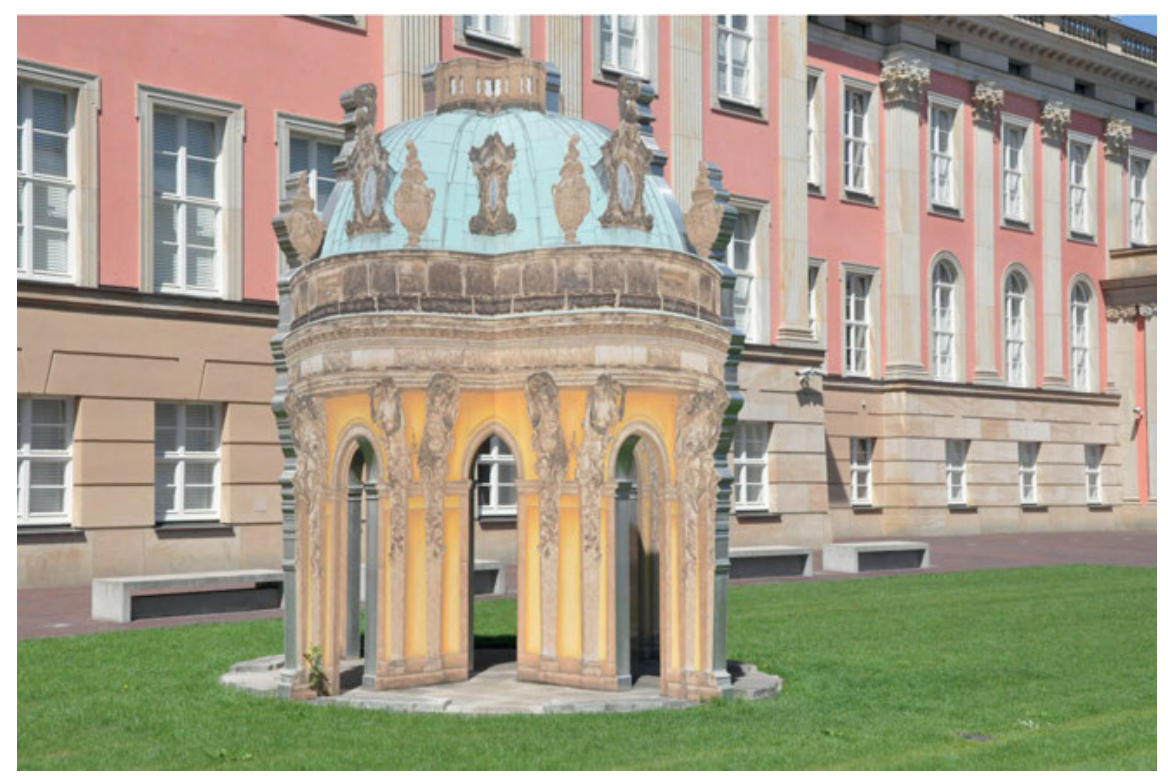

Abb. 6: Florian Dombois: Zugabe, 2014. Detail. Fotografie der Autorin, Juni 2017.

Vorder- und Rückseite, die bis auf einen Fall erfolgte Eliminierung des Schriftzuges „Sanssouci“ (denn - so der Künstler: die Pavillons sind eben nicht „Sanssouci“: „es gibt das motiv insgesamt 8 mal, aber keines ist doppelt. denn die schmalseite ist gestaucht, die rückseite gespiegelt und der zweite pavillion ja kleiner. das ,sans souci‘ hatte ich gelöscht. aber dann habe ich es doch genau einmal platziert, schön mittig zerschnitten.“ [Dombois 2017]) und durch den Umstand, dass die Pavillons eine Breitseite und eine Schmalseite aufweisen, wurde erforderlich, dass Dombois für jede dieser Malereien eine individuelle Vorlage digital erstellte. Ausdrucke dessen dienten den Malern als Orientierung. Wichtig war Dombois dabei, die Materialität der Darstellung erkennbar werden zu lassen, sodass man mit der Annäherung an die Pavillons zunächst eine Fotoästhetik erkennt, in einem Abstand von etwa fünf Metern eine Malerei und nahe am Objekt dann auch handwerkliche Verfertigung mit Unterzeichnung ausmachen kann. Beim Maler Franz Gertsch konnte er sich abschauen, wie ein malerisch zu evozierender Fotorealismus zu bewerkstelligen ist, und wandte auf seine gestaucht-gespiegelten Fotovorlagen diverse Malfilter im Programm Photoshop an, um den beauftragten Malern $\mathrm{zu}$ verdeutlichen, was eigentlich nachgeahmt werden soll: Nicht Sanssouci sollte gemalt werden, sondern das Foto davon: „wir malen fuji“ (Dombois 2017, unpag.), so Dombois. 
Die Größe der beiden Pavillons erlaubt, dass man sich durch die freigelassenen Durchgänge hindurchzwängen kann. Man nähert sich an und geht im nächsten Schritt praktisch nahtlos dazu über, sich zu entfernen. Anders ausgedrückt: Wenn man hineingeht, ist man schon wieder draußen. Die besondere Bauweise schafft keinen Innenraum. Dombois, bewandert in den Konstruktionstechniken von digitalen Welten und im Speziellen von Google Earth, betont die Basisoperation der Darstellung, die er übernimmt und für den Realraum anpasst: In der computerbasierten Modellierung „werden sämtliche plastische Körper als Volumen realisiert, über die eine zweidimensionale Textur gezogen wird“ (Dombois 2015, 11). Diese Renderingtechnik, bei der zweidimensionale Bilder über dreidimensionale Umgebungen als Texturen einer Fläche im Raum eingebunden werden, nennt sich billboarding. Die Billboards oder Z-Sprites richten sich nach dem ,Blick'-Winkel der virtuellen Kamera aus, um die Illusion des Gesamteindrucks - insbesondere bei Vegetation - zu erhöhen. Da dieses Rotieren beim Kameraschwenk aus der Nähe in aller Regel ungewollt ersichtlich wird, findet diese sparsame Lösung bevorzugt für den Mittel- und Hintergrund Anwendung (Bohnenberger undat.). In Zugabe wird die technische Lösung der Hintergrundsbefüllung nun zum Hauptgegenstand und soll zudem noch ,begehbar' sein, was sonst nur in der Entfernung für die Sichtbarkeit gedacht war. Im Innenhof des Schlosses gibt es keine Fernsicht und seine gebauten double billboards drehen sich auch nicht. Leicht hätte man dieses Verhalten auch mit einer servil-huldigenden Geste in Verbindung bringen können, als würden sogar die Bauten es verhindern wollen, dem vorbeischreitenden Souverän ihre Rückseite zu zeigen. Wohl bedacht platzierte Dombois die Pavillons auf eine Weise, die den Übergang vom absolutistischen Herrschertum, auf das die Fassaden noch alludieren, hin zum demokratischen Parlament, das gegenwärtig das Innenleben ausmacht, vermitteln. Damit galt es eine zentralistische absolutistische Situation zu verhindern, die einer politischen Logik zuspielte, wo alles auf einen Punkt hin zentriert wäre. Daher sind die Pavillons von keinem Punkt aus ohne optischen Bruch zu sehen. Dombois ließ den Grundriss am Boden als Platten aus Postaer Sandstein auslegen und in einer leichten Erhöhung die Mauerteile des Ovals in Sanssouci andeuten. Damit ergibt sich eine Parallele wie Differenz zu Bottas San Carlo alle Quattro Fontane, der in Anspielung auf die Zeichenkonventionen den Grundriss schwarz am Boden aufmalte, was mit der Schwärzung im Aufriss korrespondiert und mit der effektiven dreidimensionalen Ausführung kontrastiert. Dombois führte die Pavillons als ca. zehn Zentimeter tiefe, aber komplett ebene Flächen aus, die von der Bausubstanz der Zweidimensionalität nur wegen ihrer Anordnung überkreuz (und der Illusionsmalerei) entfliehen. Diese Flächigkeit kontrastiert mit dem Horizontalrelief des Grundrisses. 


\section{Argumentationslinien}

\subsection{Subversion der Darstellungslogik und daran etablierter Handlungstraditionen}

Welche Handhabungen möchte man an den Architekturmodellen vornehmen? Warum stellt sich bei den künstlerischen Beispielen eine Irritation ein? Die künstlerischen Beispiele weisen eine jeweils ungewohnte Verschmelzung von Darstellungs- und Funktionskonventionen auf. Wenn man die jeweiligen Rahmen verschiebt, führt dies in aller Regel zu tendenziell negativen Einschätzungen, wohl weil diese Hybride sich nicht einfach einordnen lassen. Ihnen gingen die Umgangsformen verloren, die ursprünglich mit der jeweiligen Darstellungsnorm gegeben waren und ihnen Daseinsberechtigung garantierten. Eine Darstellungskonvention als Schräggestelltes (Eisenman), Abgehacktes (Botta), Flachgelegtes (Huyghe) und Dimensionsreduziert-Überkreuzgestecktes (Dombois) effektiv zu bauen, entledigt sich scheinbar der Nützlichkeit und produziert eine Kuriosität, etwa vergleichbar mit dem realweltlichen Antreffen von typischen Merkmalen und Artefakten aus der digitalen Domäne wie Verpixelungen (Kelly Goeller, Pixel Pour, 2008), Polygonisierung (Brody Condon, 650 Polygon John Carmac, 2004) oder Namensbeschriftungen bzw. Tagging (Aram Bartholl, WoW, 2006-2009). Die Aufrisspläne (Abb. 7) von Dombois stilisieren die Pavillons als Invasion der Critter. Eine Anspielung auf die Interventionen des französischen Street Art-Künstlers Invader, der seit 1998 die reale Welt mit Figuren aus dem Arcade-Spiel Space Invader (1978) bzw. Pac-Man (1980) versieht, ist dahingehend durchaus stimmig, dass genuine Formen der computerbasierten Welten in die Realwelt katapultiert werden. Während die Importe aus der digitalen Domäne in die reale Welt in der Medienkunstszene als ,Rematerialisierungen', ,Rückwärts-(Re)Mediatisierungen“5

5 Der Ausdruck ,backwards mediation“ ist ein synthetischer Begriff, der eine kritische Debatte rund um den vom Künstler James Bridle 2011 ins Leben gerufenen Terminus ,New Aesthetics‘ zusammenfasst. Ungeachtet aller möglicher philosophischer Konnotationen sollte dieser Ausdruck lediglich dazu dienen, seiner Tumblr-Sammlung an digital-informierten Artefakten ein Motto zu verleihen. Die von Bridle damit bezeichneten und zusammengetragenen Phänomene verkörpern laut den Medientheoretikern Jay David Bolter und Richard Grusin nichts anderes als ihr eigenes funktionales Konzept der Remediatisierung, wobei für Grusin Bridles Form der „remediation“ eine aufgeplusterte Variante sei, die nirgendwohin führe (vgl. Grusin 2012; Bolter und Grusin 1999). Dies kommentierend, spricht die Kunsthistorikerin Katja Kwastek von einer „backwards mediation, from the digital back to the analogue, from the virtual back to the physical, abstracting a visual language from its technological conditions“ (Kwastek 2015, 75). Es wird Bridles technologisch-digital informierte Ästhetik durchaus Relevanz zuerkannt, aber das Gemeinte decke schon ein anderer Begriff ab, den Kim Cascone 2002 in die Musikologie eingeführt hat, nämlich 


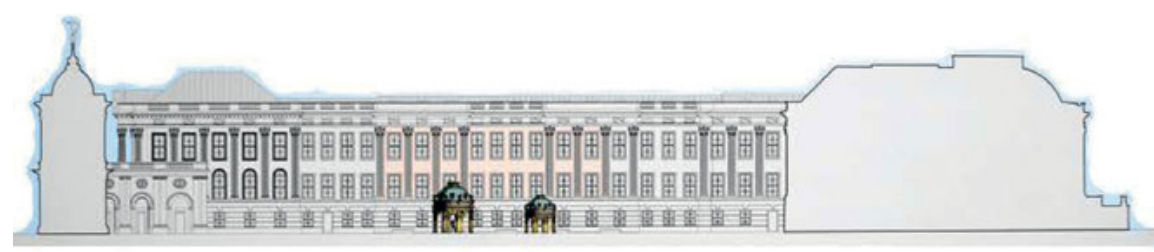

Abb. 7: Florian Dombois: Zugabe, 2014. Positionierung der Pavillons im Aufriss. Zeichnung aus der Phase 1 des Wettbewerbs, 2012. Quelle: Mit freundlicher Genehmigung des Künstlers.

oder ,postdigital' bezeichnet werden, fehlt meines Wissens die Begrifflichkeit für die Überführung aus der zweidimensionalen Domäne. ,Verräumlichung‘ allein kann die Irritation noch nicht erklären. Die gezeigten Beispiele referieren alle auf existierende Bauten oder sind zumindest post factum entstanden. Sind es deren Rekonstruktionen? Genau genommen sind es Bauten nicht nach, sondern von Plänen bzw. Darstellungskonventionen. Die Architekten und Künstler haben eine selbst gewählte Vorlage bewusst ,wörtlich` bzw. ,bildlich` genommen und kamen zu Lösungen, die Zweidimensionalität verkörpern und dennoch keine unmittelbar zutage tretenden, üblichen Handlungsräume an ihnen eröffnen. Bei Eisenman könnte man nur manche der Längen messen, bei Bottas XXL-Modell ist man als Betrachter_in plötzlich miniaturisiert, wodurch ein Überblick nur mehr aus großer Entfernung gelingt und dann ist es mit dem bequemen Messen vorbei. Bei Huyghe lässt das flache Kantenmodell wie bei einem am Boden liegenden vielgliedrigen Mobile noch nichts erkennen, erst wenn es hochgezogen wird, entfaltet sich das wackelige Konstrukt. Bei Dombois' Pavillons kann die konkave Räumlichkeit nur gesehen werden, nie aber funktioniert diese Illusion für beide zugleich. Die Irritation, die diese Beispiele hervorrufen mögen, zeigt, dass die Gestaltung der Modelle, die immer auch eine Umgangstradition mitführen, einen wesentlichen Einfluss darauf nehmen, was an ihnen sinnvoll getan werden kann. Sie verweigern sich durch ihren besonderen Brückenschlag zum Bildmaterial, auf dem sie basieren, manchen Umgangspraxen. In ihrer künstlerischen Attitüde verletzen sie kulturell gewachsene Regeln.

das ,post-digital‘ (vgl. Cascone 2002). Das Post-Digitale betont nach dem Medientheoretiker Florian Cramer einen Desillusionismus und meint auch eine tiefe Verschränkung digitaler Technologie im Alltagsleben: „[. . .] today, digital technology is deeply embedded in ,everyday life‘. It serves to emphasize that the ,digital' is not as definite as we might assume: that it is no ,virtual reality" distinct from our everyday world, but a constitutive part of it“ (Kwastek 2015, 79). 


\subsection{Modelle als Affordanzen: poetogen und praxigen?}

Die vorgestellten architektonischen und künstlerischen Beispiele, bei denen sich eine räumliche Konfiguration mit der Logik der Axonometrie, des Aufrisses, der Aufführung und der Billboards mischt, scheinen sich gerade einem sinnvollen oder zumindest gewohnten Umgang zu sperren. Dafür bieten sie etwas Anderes. Sie erweisen sich als betont und bewusst fotogen oder im Falle von Huyghes Arbeit auch als telegen. Sie sind vielleicht sogar bildseriengemäß, denn sie alle bereiten potenziell eine Verblüffung rein dadurch, dass sie mehr als einen Blickpunkt erlauben, während die Darstellungslogiken, auf denen sie basieren, diese Varianz gerade suspendieren. Der Kulturwissenschaftler Ulrich Raulff deutet für das Wort ,fotogen' den Übergang vom Bild(schöpfungsverfahren) auf den Referenten als ein prothetisches Wirkungsverhältnis an (Raulff 1986, 50, 53 und 56). Philipp Weiss führt dazu näher aus:

,Fotogen', das bedeutete ursprünglich schlicht: mit Mitteln der Fotografie erzeugt, fotogeneriert. Fotogen aber meint nun den Referenten, der sich besonders gut eignet, fotografisch repräsentiert zu werden, der auf Fotos ,gut kommt‘. [. . .] Nicht jeder ,gutaussehende‘ Mensch ist auch fotogen, nicht jeder Fotogene überzeugt auch noch als unmittelbare Erscheinung oder filmische Reproduktion (telegen, besser cinegen) unsere Augen. Und diese Differenz bedeutet auch: ein Foto muss nach bestimmten Regeln hergestellt werden, um eine befriedigende oder erfüllende Repräsentation eines Subjektes zu schaffen. Es ist jetzt vielleicht an der Zeit zu fragen, wie das Foto das Feld des Visuellen bestimmt und was es tut [. . .] wenn es einen Körper repräsentiert. [meine Hervorhebung, I.H.].

(Weiss 2010, 101)

Analog ist hier $\mathrm{zu}$ fragen: Wie bestimmt eine 3D-Rekonstruktion das Feld der Handlungen und was tut sie, wenn sie $\mathrm{Zu}$ - und Eingriffe erlaubt? Wenn bildliche Projektionen auf den Visus ausgerichtet sind, ginge es nun darum, eine Regel zu suchen bzw. zu bestimmen, wie man verschiedene Dinge behandelt? Perspektiviert man Handlungen dadurch, dass man eine Konfiguration erstellt, die Zugangsbedingungen verkörpert und dadurch nur ganz bestimmte Eingriffe erlaubt (handlungsbezogene Grenzziehungen)? Braucht es dazu immer schon ein zwischengeschaltetes Interface? Wenn in Modellen als repräsentierende Werkzeuge Handlungen in den Vordergrund gestellt (antizipiert und optimiert) werden, geht es weniger um das ,Fotogene' und mehr um das Operative. Wie wird ein Modell für ein zweckgebundenes, gezielt-erreichbar-abschließbares Handeln ,poetogen“ und für ein freieres Handeln zum Selbstzweck ,praxigen`? ${ }^{6}$ Braucht es dazu eine Tradition?

6 An dieser Stelle ein Dank an Michail Chatzidakis, der mich auf mögliche Fallstricke bei diesen Wortschöpfungen aufmerksam machte. 


\subsection{Möglichkeit statt Virtualität - philosophische Seinsweisen vertauscht}

Man könnte es aber auch so interpretieren, dass die Irritation dadurch zustande kommt, dass man die Vorlagen als Möglichkeiten realisierte und nicht als Virtualitäten aktualisierte. Was bedeutet das? Die Philosophen Gilles Deleuze, Pierre Lévy und Brian Massumi verstehen das Virtuelle als Seinsweise, die sich vom Möglichen, Potenziellen, Aktuellen absetzt, der aber durchaus eine Realität im Virtuell-Sein zuzugestehen ist. Das Virtuelle lasse sich nur induktiv über die Effekte seiner Aktualisierung erspüren. Es habe im Unterschied zum Digitalen ein Moment des flüchtigen Aufscheinens, lässt sich dennoch aber nicht direkt erblicken, sondern wird über die Variationsbreite seiner Ausprägungen und als deren Eingefaltetes evident. Weil die Topologie die Lehre der selbstvariierenden Deformation sei, sei sie laut Massumi der einzige Weg, über den sich die Imagination dem Virtuellen annähern könne. Andernfalls bleibt man allzuleicht bei anderen Daseinsweisen hängen:

you miss the virtual unless you carry the images constructed in that medium to the point of topological transformation. If you fall short of the topological, you will still grasp the possible (the differences in content and form considered as organizable alternatives). You might even grasp the potential (the tension between materially superposed possibilities and the advent of the new). But never will you come close to the virtual.

(Massumi 2002, 133-134)

Alle Künste und Technologien können ein Virtuelles eingeschlossen halten, was gegeben ist, wenn sie einen Nukleus der qualitativen differenziellen Transformation aufweisen. Man nehme dabei in Acht, das Diaphane des Virtuellen nicht mit dem Artifiziellen des Digitalen zu verwechseln: „Nothing is more destructive for the thinking and imaging of the virtual than equating it with the digital“ (Massumi 2002, 137). Das computerbasierte Technisch-Digitale verführe gerne zum fehlgeleiteten Fokus, da es in der Umsetzung des Möglichen so mächtig sei. Für Lévy ist das Mögliche ein vollständig vorgefertigtes Latentes, das parat steht, um in die Realität gebracht zu werden (was auch ein Automat vollbringen könnte). Im Unterschied dazu benötigt die Umsetzung des Virtuellen eine kreative Leistung, bringt neue Qualitäten und Ideen hervor, die sich abermals ans Virtuelle zurückspeisen können. „Unlike the possible, which is static and already constituted, the virtual is a kind of problematic complex, the knot of tendencies or forces that accompanies a situation, event, object, or entity, and which invokes a process of resolution: actualization. This problematic complex belongs to the entity in question and even constitutes one of its primary dimensions“ (Lévy 1998, 24). Und weiter schreibt er: „The virtual [. . .] is a 
fecund and powerful mode of being that expands the process of creation, opens up the future, injects a core of meaning beneath the platitude of immediate physical presence“ (Lévy 1998, 16). Wenn nun die Aktualisierung des Virtuellen einen Akt der Problemlösung darstellt, welches Problem wollen die architektonischen und künstlerischen Beispiele lösen? Keines, so die These. Aus meiner Sicht wurde hier tatsächlich keine Lösung eines Problems in Angriff genommen, obwohl die diskutierten Artefakte der Domäne der Modelle zuordenbar sind, welche für Problemlösungsstrategien prädestiniert wären. Stattdessen entschieden sich die Kunstschaffenden bewusst, dem Seinsmodus der Möglichkeit Ausdruck zu verschaffen, wofür man auch im Mimetischen einen Anhaltspunkt finden könnte (Sie haben die Vorlagen als Möglichkeiten wiedererkennbar realisiert.): So schreibt Lévy: „The real resembles the possible. The actual, however, in no way resembles the virtual. It responds to it“ (Lévy 1998, 25).

\section{Akademische Modellierungen}

Digitale Rekonstruktionen reihe ich in die Traditionslinie der Modelle. Sie sind insofern komplementär zu den besprochenen Beispielen, als sie Analoges in die digitale Domäne bringen. Das kapselt sie aber nicht vom Virtuellen ab. Im Gegenteil, man darf die wichtige epistemische Frage der Handlungsperspektivierung, welche die 3D-Rekonstruktionen leisten und die nichts anderes sind als die Spezifik des Virtuellen, nicht vergessen. Die Präsentation neuer Handlungsgelegenheiten (Verschiebung wie Skalierung, Beschleunigung etc.) aber sei wiederum ein erster Grad an Virtualisierung. Die Virtualisierung sei nach Lévy die umgekehrte Bewegung der Aktualisierung (Lévy 1998, 26). Massumi nennt diese Bewegung, die über eine Aktualisierung hinausgeht und sie anreichert „Deactualization“ (Massumi 2002, 136).

\subsection{Epistemologische Kategorie der Computermodelle als Komposite}

Im Jahr 2014 präsentierte der Systembiologe Sol Efroni von der Bar Ilan University in Israel auf der Konferenz „From in vitro to in vivo“ in Lüneburg das Ergebnis eines Kraftaktes (Efroni 2014; Efroni et al. 2007). Er hatte 400 Publikationen zum Thema der Thymus-Lymphozyten auf Ergebnisse abgeklopft. Die Forscher führten die kleinen Bausteine des verstreuten Wissens über die T-Zellen über so genannte animierte state charts als ausführbare ,Geschichten“ 
zusammen. Mittels einer Zoomfunktion ließen sich Metadaten abfragen und Vorgänge auf unterschiedlichen Beschreibungsebenen einsehen. Es stellte sich dabei heraus, dass keine Publikation die Dimension der Zeit berücksichtigte, was dadurch sofort ins Auge sprang, weil alle diese Erkenntnisse in eine einzige interaktive, zeitbasierte Simulation integriert werden sollten. Diese Zusammenführung gelang laut Efroni weitestgehend. Lediglich einige wenige Vorschläge divergierten derart vom restlichen Corpus, dass sie nicht integriert werden konnten und ergo unplausibel waren. Diese Schlussfolgerung trat in der Forschungsgruppe „Medienkulturen der Computersimulation“ sodann die Diskussion los, wie man aufgrund dieser Argumentation Computersimulationen einzuschätzen hat: als eine Art Übersichtsartikel, der programmtechnisch ausgeführt wird (,review article that is running'); oder gar als eine neue epistemologische Kategorie zwischen Theorie und Experiment?

Mit Blick auf die 3D-Rekonstruktionen von Gebäuden scheint sich zuweilen Vergleichbares einstellen zu können. Zum einen mündet eine faszinierende Vielfalt an Quellen in eine Architekturrekonstruktion, die ihr mehr ,Substanz und Bewusstsein dafür, was fehlt, sowie einen besonderen Stellenwert verleiht, denn zum anderen taucht die Idee des Experiments auch in diesem Anwendungsfeld auf. Hierzu zitiere ich Heike Messemer, die 2014 in Bezug auf die digitalen Visualisierungen schrieb:

Während der Arbeit an solchen digitalen Visualisierungen ergeben sich für die Wissen-
schaftler immer wieder neue Fragen: Lücken in den Quellen, widersprüchliche Aussagen
in Überlieferungen oder neue bauphysikalische Befunde gilt es in digitale Visualisierun-
gen erkenntnisorientiert einzubringen. Hypothesen können erprobt und wieder verworfen
werden, frühere Interpretationen relativiert oder bestätigt werden. Denn ein Modell birgt
den nicht zu unterschätzenden Vorteil, Überlegungen und Ideen bildhaft zu veranschau-
lichen. Ob eine Hypothese funktioniert oder nicht, ist in der Verbildlichung oft leichter zu
erkennen als allein in der schriftlichen Reflexion.
(Messemer 2014, 15)

Auch ließen Severin Todt und Kollegen 2009 verlauten: „Erstmals stand den Historikern damit [mit den digitalen Ansichten eines groben 3D-Modells] eine visuelle Möglichkeit zur Verfügung, historische Daten einer Richtigkeitsprüfung zu unterziehen“ (Todt et al. 2009, 124). Laut der Architekturhistorikerin Diane Favro ermuntern die ,Rekreationen' als Wissensrepräsentationen auf diversen Ebenen eine Benutzung. Somit funktionieren sie wie Laboratorien, in denen Spezialist_innen gegenstandsbezogene Annahmen zerlegen und austesten können (Favro 2006, 327).

Wenn dem so ist und weil computerbasierte geometrische Modelle keine Simulationen (verstanden als gerechnete kybernetisch-formalisierte Dynamik) sind, scheint mir stärker noch als die Kulturtechnik der Computersimulation 
die Logik der Assemblage, des Clusterns, des Komposits für diesen bemerkenswerten Status ausschlaggebend zu sein, die aber weniger der Geste des paritätisch-kombinatorischen Nebeneinander und mehr jener des verzahnenden Ineinander entspricht. Möglicherweise liegt es daran, dass eine zunächst fragile Anhäufung an Mutmaßungen, Partialkonsistenzen und interdisziplinären Zugriffen das Potenzial akkumuliert, um irgendwann als eine ,Instanz“ wahrgenommen werden zu können. Der Wissenschaftstheoretiker Ian Hacking spricht in diesem Zusammenhang von „robusten Fugungen“: „Die Fugung zwischen Theorie, Phänomenologie, schematischem Modell und Geräten ist dann robust, wenn bei Versuchen der Wiederholung eines Experiments keine Schwierigkeiten auftreten und wenn andere Forschergruppen mit neuen Geräten, neuem latentem Wissen und anderer Experimentierkultur auf keine neuen Widerstände von Gewicht stoßen“ (Hacking 1999, 113; vgl. ferner Hinterwaldner und Buschhaus 2006). Auf diese Weise könne, so Hacking, aus einem ,vorläufigen Haltepunkt‘ ein ,bleibender Bezugspunkt' werden. Differenzierter schildert William Wimsatt diese Wende im Forschungsprozess, an der das epistemische Ding vom zu Stabilisierenden ins zu Verteidigende umschwenkt, das gar als Instanz des Abgleichs für alle später dazukommenden Daten dient (Wimsatt 1994, 214-215). ,Robust‘, ,stabil` oder ,solide‘ wird eine Entität genannt, die sich dadurch plausibel herauskristallisiert, sodass es gelingt, unterschiedlichste dafür evidenzstiftende Elemente zusammenzufügen. Der Fugungsmodus ist nicht ein Konzept des Einflusses, sondern der Konfluenz.

Freilich ist die Idee des Zusammenfließens, der Synthese, angesichts der Notwendigkeit, bei einer VR-Rekonstruktion viele Weichen vorab definieren zu müssen, nicht unterkomplex anzulegen. Wenn man bedenkt, was alles Berücksichtigung findet, kommen die vielen verschiedenen Filter zum Vorschein, durch die man auf das zu Rekonstruierende zublickt und zugreift. Je nach Zielsetzung und Fragestellung, je nach Gewissheitsgrad der Unterlagen, je nach Zielpublikum, je nach Fachkultur und der darin prävalenten ,professional vision' (um mit dem Linguisten Charles Goodwin zu sprechen), wird das zu Rekonstruierende anders dargestellt. Laut Favro nehmen die unterschiedlichen disziplinären Präferenzen im Schauen in der Art, der Präsentation und dem Status von Illustrationen in der jeweiligen Fachkultur ihren Ausgang. Kunsthistoriker_innen würden aus ihrer Erfahrung gerne am Architekturmodell ,hochfliegen', um Details wie Giebelreliefs auf Augenhöhe zu studieren und sie dann gemäß den Blickwinkeln zu rekonstruieren, die über Fotografien belegt sind. Die Altphilolog_innen hingegen wollen nachvollziehen, wie es sich anfühlt, auf der antiken Rednerbühne gegenüber dem Senatsgebäude der Stadt Rom zu stehen (Favro 2006, 333 und Fußnote 61). Die Bauforscher_innen verfolgen „üblicherweise die Absicht, ein dreidimensionales Objekt in seinem Bestand 
verformungsgerecht $\mathrm{zu}$ dokumentieren und seine konstruktiven Zusammenhänge und bauhistorische Entwicklung aufzuzeigen. Das Modellierungsanliegen von Vermessungskundlern hingegen liegt vorrangig in einer möglichst exakten Wiedergabe der Objektgeometrie und seiner Oberflächen“ (Verstegen 2007, 10). Architekt_innen bevorzugen normalerweise einen unverstellten Blick auf das Gebäude, haben aber durchaus eine Affinität für „Wege-, Blick- und Lichtführung“ (Breitling 2012, 28). Archäolog_innen fokussieren mehr auf den gesicherten Einzelbefund und halten sich „bei der Raumdarstellung eher zurück“ (Breitling 2012, 28), interessieren sich jedoch auch für den jeweiligen Nutzungszusammenhang. Modelle verkörpern Interessen, Sichtweisen und Strategien, aber auch - so die These - Virtualität in Bezug auf die Handlungsweisen. Unter diesem Vorzeichen ist es fast utopisch, dass es gelingt, in eine einzige digitale Rekonstruktion alle disziplinären Vorlieben zu integrieren. Gelingt dies trotz alledem, hat man es - epistemologisch - mit einer besonders robust gefugten Entität zu tun. Derselben Herausforderung wird auch Efroni gegenübergestanden haben, weswegen er imstande gewesen sein müsste, in seiner Simulation auch mehrere Beschreibungsebenen zu integrieren.

Sollte man von einer Einheitlichkeit im Zugang auf das (so) nicht mehr existierende und daher digital rekonstruierte Gebäude absehen? Wäre es - in Anbetracht der verschiedenen beteiligten Disziplinen und deren spezifischen Blicke (Goodwin 1994; Styhre 2010) - wünschenswert, dass Modelle als Wissensobjekte mannigfaltige Zugänge erlauben? Wäre denkbar, dass die Kunsthistorikerin Sarada Natarajan ihre experimentelle Methodik der ausdifferenzierten Herangehensweisen im Prinzip auch in virtuo ausführen könnte? Natarajan definierte bei ihrer Feldforschung in Ravana ki Khai, der Höhle 14 in Ellora, Indien, bestimmte Rollen, um sich wiederholt derselben Höhle zu nähern. Sie ging jeden Tag bewusst mit einer anderen Haltung, Aufgabenstellung samt entsprechenden Instrumenten, in die Höhle: einmal als Fotografin, beim zweiten Mal als Zeichnerin, dann als Vermessende (mit Maßstab und mit Körpergröße), als sprechend Beschreibende und schließlich als schriftlich Notierende. Der Untersuchungsgegenstand zeigte sich aufgrund der Fokusverschiebungen auf unterschiedliche Weise und ließ sich auf vielfältige Weise befragen (Natarajan 2017). Dieses Phänomen könnte man mit dem Philosophen Ludwig Wittgenstein diskutieren, für den das Denken zur Wahrnehmung hinzutritt, wenn der Sinneseindruck mal als dieses und mal als jenes interpretiert wird, d. h., wenn man die Änderung wie eine Wahrnehmung beschreibt, „ganz als hätte sich der Gegenstand vor meinen Augen gewandelt“ (Wittgenstein 1984a, § 476), wobei sich aber unbegreiflicherweise „nichts geändert hat, und sich doch Alles geändert hat“ (Wittgenstein 1984b, § 474). Für Wittgenstein 
zeigt sich „das Beteiligtsein des Denkens genau beim Wechsel des Aspektes, just in dem Augenblick, in dem sich das wirkliche Umkippen vollzieht, denn diesem Umschalten entspricht ja keinerlei Pendant in einem ,Umkippen der Zeichnung selbst‘ [. . .]“ (Krämer 2009, 116). Dies führt zurück zur Frage nach epistemischen Dimensionen dieser hochkomplexen, spannenden und spannungsgeladenen digitalen Wissensräume. Passiert es automatisch, dass für jede Disziplin, die an eine 3D-Rekonstruktion Fragen heranträgt, oder für jede Forscher_in, das Modell sich anders zeigt? Oder kann man diese Fähigkeit beim Modellbau forcieren?

\subsection{Auf der Suche nach Kategorien für digitale 3D-Rekonstruktionen}

Sehr grundlegend gilt für Modelle, dass sie manche Eigenschaften des Originals aufweisen, darüber hinaus aber noch Merkmale besitzen, die sie damit nicht teilen (vgl. Verstegen 2007, 9). Im Kontext der digitalen Rekonstruktion von Kulturgütern ist naheliegend, dass genuin für das Modell bleibt, wie mit ihm umgegangen werden kann. Die Suche nach handlungsbezogenen Vorbildern bei digitalen Rekonstruktionen und - das hängt damit zusammen - auf welche Weisen das computer-basierte Modell aufgefasst wird, ist im Gange. Anleihen werden zunächst bei bekannten Konzepten genommen. In einschlägigen Publikationen liest man beispielsweise, das 3D-Modell sei als browsebares Interface (Apollonio et al. 2013, 43) aufzufassen, oder als natürlicher visueller Index zu historischen bzw. künstlerischen Informationen, die durch so genannte ,hot spots“ (Baracchini et al. 2004, 149) zugänglich gemacht werden; oder als Instanz, in die neue Erkenntnisse zügig ,eingepflegt‘ werden können (Verstegen 2007), oder „als Zugangsoberfläche zu verschiedenen Datenbanken, Managementsystemen, als Findbuch für Inventare und Aktenbeständen etc.“ (Breitling 2012, 31).

Fasst man die Modelle als gestaltete Artefakte auf, die einerseits aspektiv sind und andererseits nicht zuletzt dadurch Affordanzen (intentionsgesättigte Handlungsangebote) bieten, so könnte man obige Aussagen dahingehend zusammenfassen, dass sie - im Unterschied zur Architektur selbst - primär eine Schnittelle sind, die Informationen motivisch-situativ in den dreidimensionalen Raum abrufbar einbettet. Manche gehen sogar so weit zu sagen, ein wissenschaftlicher Nutzen sei erst über die Metadaten gegeben, die die zugrundeliegenden Thesen nachvollziehbar werden lassen. Dafür steht der Begriff der ,transparenten Grundlagenforschung، (Engel und Guminski 2016, 80). Diese Transparenz scheint jedoch beinahe das ikonische Komposit als Zusammengeführtes zum Verschwinden zu bringen, 
wenn es das Bild nur als Durchgangsportal nutzt. Bei vielen datenbankbasierten Arbeiten stehen die Bilder nicht im erwarteten Nahverhältnis zu den gewählten Interventionsstrategien. Der Mediengestalter Andrew Polaine charakterisiert den Typus an Interaktivität, der hier zugange ist, als einen, bei dem das Bild - in diesem Fall die am Monitor ausgegebene virtuelle 3D-Rekonstruktion - als ,Durchgang‘ zum eigentlichen Inhalt verstanden wird. Was die ,Interaktivitätals-Schleuse“ anbelangt, formulierte er pointiert: „This ignores the experience of the moment of interaction and relegates it to a mechanism of control at best and something to be mastered and ,got through" at worst“" (Polaine 2004, 733). Der russisch-amerikanische Künstler und Medientheoretiker Lev Manovich diskutiert Bilder, die sowohl eine operative als auch eine repräsentierende Komponente besitzen und unterscheidet zwischen ,Bild-Interface“ (image-interface) und ,Bild-Instrument‘ (image-instrument). Während man das Bild-Interface dafür nutzt, im Computer etwas $\mathrm{zu}$ bewirken, wird das BildInstrument dafür eingesetzt, in die Realität $\mathrm{zu}$ affizieren (Manovich 2001, 183). Ob man dies wirklich so genau separieren kann, sei an dieser Stelle dahingestellt. Jedoch scheinen etliche der oben zitierten Charakterisierungen die Rekonstruktion als Bild-Interface aufzufassen.

Während das Paradigma der Human-Computer Interaction als Gestaltungsdomäne der Schnittstelle zunächst zu einem Desktopcomputer - heute auch zu mobileren Geräten - hinlänglich bekannt ist, scheint es hier jedoch schon stärker um ein architektur- bzw. bauspezifisches Setting zu gehen, als nur um eine multifunktionale Schnittstelle zum Rechner. Auch dafür gibt es einen etablierten Ansatz: Für den Ingenieur Hamed S. Alavi und Kolleg_innen besteht die Gestaltung der Human-Building Interaction (HBI) darin, den Bewohner_innen interaktive Gelegenheiten $\mathrm{zu}$ bieten, die physikalischen, räumlichen und sozialen Auswirkungen ihrer gebauten Umgebung zu formen (Alavi et al. 2016). Beim Versuch, dieses Konzept auf die digitalen Rekonstruktionen zu übertragen, und damit mit HCI zusammenzuführen, ist darauf zu achten, dass die Gestaltung der Human-DigitalBuilding Interaction (HDBI) weniger das Bewohnen und mehr das Explorieren im Fokus haben wird. Es ginge jedoch auch um motivbezogene - d. h. auf Bauten hin orientierte - Umgangsweisen. Welche Handlungen will man an den Architekturmodellen vornehmen (bzw. als Gestalter_innen daran vornehmen lassen), die man an der Architektur selber nicht vornehmen kann? In der Palette des Vollführbaren scheint das Virtuelle auf. Kann man es gestalten? Ist das dasselbe wie ,virtualisieren'? Wo trifft sich das Virtuelle mit dem Interaktionsdesign? 


\section{Literatur}

Alavi, Hamed S., Denis Lalanne, Julien Nembrini, Elizabeth Churchill, David Kirk und Wendy Moncur. „Future of Human-Building Interaction“. Proceedings of the $2016 \mathrm{CHI}$ Conference Extended Abstracts on Human Factors in Computing Systems (CHI EA '16) (2016). 3408-3414. Apollonio, Fabrizio I., Marco Gaiani und Zixiang Sun. „3D modeling and data enrichment in digital reconstruction of architectural heritage". International archives of the Photogrammetry, Remote Sensing and Spatial Information Sciences, Vol. XL-5/W2, XXIV International CIPA Symposium, Strasbourg 2.-6. September 2013, (2013). 43-48.

Baracchini, Clara, Anntonio Brogi, Marco Callieri, Lucia Capitani, Paolo Cignoni, Andrea Fasano, Claudio Montani, C. Nenci, R.P. Novello, Pingi, Paolo, Federico Ponchio und Roberto Scopigno. „Digital reconstruction of the Arrigo VII funerary complex“. 5th International Symposium on Virtual Reality, Archaeology and cultural Heritage (VAST) (2004). 145-154.

Bellini, Rolando. „Ifetecne del San Carlino sul lago di Lugano“. Mario Botta per Borromini: il San Carlino sul lago di Lugano. Hg. Ders. und Fabio Minazzi. Varese: Edizioni Agorà, 2000. 11-49.

Bertelli, Carlo. „Francesco Mario „Bottomini‘, ovvero Borromini sul lago“. Borromini sul lago. Mario Botta: La rappresentazione lignea del San Carlo alle Quattro Fontane a Lugano. Ausst.Kat. Museo Cantonale d'Arte, 4. September - 14. November 1999. Hg. Gabriele Cappellato. Genf, Mailand, Mendrisio: Skira, Accademia di architettura, 1999. 21-23.

Bohnenberger, Marcel. Billboarding. http://mbohnenberger.com/billboarding.html (9. Juni 2017).

Bolter, David und Richard Grusin. Remediation. Understanding New Media. Cambridge, MA: MIT Press, 1999.

Botta, Mario. „Appunti sulla rappresentazione lignea del San Carlino a Lugano“. Borromini sul lago. Mario Botta: La rappresentazione lignea del San Carlo alle Quattro Fontane a Lugano. Ausst.Kat. Museo Cantonale d'Arte, 4. September - 14. November 1999. Hg. von Gabriele Cappellato. Genf, Mailand, Mendrisio: Skira und Accademia di architettura, 1999. 13-19.

Breitling, Stefan. „Raum und Zeit im digitalen 4D-Modell - Virtuelle Architekturen als dynamische Wissensräume“. Elektronische Medien \& Kunst, Kultur, Historie, Proceedings. Hg. Bienert, Andreas, Frank Weckend und James Hemsley. Berlin 7.-9. November 2012 (EVA 2012). Berlin: Staatliche Museen zu Berlin und Gesellschaft zur Förderung Angewandter Informatik e.V., 2012. 26-32.

Cappellato, Gabriele (Hg.). Borromini sul lago. Mario Botta: La rappresentazione lignea del San Carlo alle Quattro Fontane a Lugano. Ausst.Kat. Museo Cantonale d'Arte, 4. September - 14. November 1999. Genf, Mailand und Mendrisio: Skira und Accademia di architettura, 1999.

Cascone, Kim. „The Aesthetics of Failure: ,Post-Digital‘ Tendencies in Contemporary Computer Music“. Computer Music Journal 24.4 (2002). 12-18.

Dombois, Florian. Zugabe. Konzeptbeschreibung, 2012.

Dombois, Florian. „Zugabe. Auszug aus der Bewerbung zum Wettbewerb“. Kunst am Bau für den Landtag Brandenburg, Potsdam. Hg. Landtag Brandenburg. Potsdam, 2015. 11-13. Dombois, Florian. Persönliche E-Mailkorrespondenz, 4. Juni 2017. 
Efroni, Sol. „Compiling and Running Biological Observations as 3D Computer

Experimentation“. Vortrag, 21. Juni 2014 auf der Konferenz From in vitro to in silico.

Computer Simulation in the Life Sciences. MECS, Lüneburg.

Efroni, Sol, David Harel und Irun R. Cohen. „Emergent Dynamics of Thymocyte Development and Lineage Determination“. PLoS Computational Biology 3.1 (2007) e13. https://doi.org/ 10.1371/journal.pcbi.0030013 (20. April 2019).

Eisenman, Peter. House X. New York: Rizzoli, 1983.

Elber, Gershon. Escher for Real. http://www.cs.technion.ac.il/ gershon/EscherForReal/ (4. Mai 2014).

Emery, Nicola. „Volontà d'arte“. Borromini sul lago. Mario Botta: La rappresentazione lignea del San Carlo alle Quattro Fontane a Lugano. Ausst.Kat. Museo Cantonale d'Arte, 4. September - 14. November 1999. Hg. von Gabriele Cappellato. Genf, Mailand, Mendrisio: Skira und Accademia di architettura, 1999. 39-43.

Engel, Ute und Karin Guminski. „Die Kammerkapelle der Kurfürstin in 3D“. Akademie Aktuell 2 (2016). 77-81.

Favro, Diane. „In the eyes of the beholder. Virtual Reality re-creations and academia“. Imaging ancient Rome. Documentation, visualization, imagination. Hg. von Lothar Haselberger und John Humphrey. Proceedings of the 3rd Williams Symposium on Classical Architecture, Rom 20.-23. Mai 2004; zugleich Journal of Roman Archaeology Band? (2006). 321-334.

Friedberg, Anne. The Virtual Window. From Alberti to Microsoft. Cambridge, MA und London: MIT Press, 2006.

Goodwin, Charles. „Professional Vision“. American Anthropologist 96.3 (1994). 606-633.

Grusin, Richard. „The ,new aesthetic“ is Just the Latest Name for Remediation, All Dressed Up with Nowhere to Go“. Twitter, 18. April 2012. https://twitter.com/rgrusin/status/ 192622844860047361 [20. 04.2019].

Hacking, lan. Was heißt, soziale Konstruktion‘?. Frankfurt am Main: Fischer TB, 1999.

Hinterwaldner, Inge und Markus Buschhaus. „Zwischen Picture und Image. Überlegungen zu einem komplementären Kompositbegriff“. The Picture's Image. Wissenschaftliche Visualisierung als Komposit. Hg. dies. und ders. München: Fink, 2006. 9-19.

Hinterwaldner, Inge. „Künstlerisches und architektonisches Entwerfen - , not a time for dreaming'?".). Bildlichkeit im Zeitalter der Modellierung. Operative Artefakte in Entwurfsprozessen der Architektur und des Ingenieurwesens. Hg. Ammon, Sabine und dies. München: Fink, 2017. 315-345.

Jäger, Heidi. „Sanssouci, zerschnitten“. Potsdamer Neueste Nachrichten. 6. September 2012. http://www.pnn.de/potsdam-kultur/678649/ (29. Mai 2017).

Krämer, Sybille. „Operative Bildlichkeit. Von der >Grammatologie< zu einer $>$ Diagrammatologie<? reflexionen über erkennendes >Sehen<“. Logik des Bildlichen. Zur Kritik der ikonischen Vernunft. Hg. Heßler, Martina und Dieter Mersch. Bielefeld: transcript, 2009. 94-123.

Kwastek, Katja. „How to be theorized: A Tediously Academic Essay on the New Aesthetic“. Postdigital Aesthetics. Art, Computation and Design. Hg. Berry, David M. und Michael Dieter. New York: Palgrave Macmillan, 2015. 72-85.

Lévy, Pierre. Becoming Virtual. Reality in the Digital Age. New York, London: Plenum Trade, 1998. 
Lordick, Daniel. „Schiefe Bilder. Mit Rapid Prototyping zu räumlichen Anamorphosen“. Tagungsband der DGfGG, (Universität Hannover 2005). 141-150. http://www.lordick.dar stellende-geometrie.de/docs/Schiefe-Bilder-Lordick.pdf (16. Dezember 2011).

Manovich, Lev. The Language of New Media Cambridge, MA und London: MIT Press, 2001. Massumi, Brian. „On the Superiority of the Analog“. Parables for the Virtual. Movement, Affekt, Senation. Durham, NC und London: Duke University Press, 2002. 133-143.

Messemer, Heike. „Architektur und Erinnerung in digitalen Welten“. profile. Magazin über Architektur 13: (2014). 14-17.

Minazzi, Fabio. „Per una lettura epistemological del San Carlino di Mario Botta. Mario Botta per Borromini: il San Carlino sul lago di Lugano. Hg. Bellini, Rolando und ders. Varese: Edizioni Agorà, 2000. 50-87.

Natarajan, Sarada. Marking Making: The vexed issue of artistic agency in Indian sculptural historiography. Vortrag im Institut für Kunst- und Bildgeschichte der HumboldtUniversität zu Berlin am 10. Mai 2017 und persönliches Gespräch am 29. Mai 2017.

Panza di Biumo, Giuseppe. „Lo spaccato di San Carlino alle Quattro Fontane di Roma a Lugano“. Borromini sul lago. Mario Botta: La rappresentazione lignea del San Carlo alle Quattro Fontane a Lugano. Ausst.Kat. Museo Cantonale d'Arte, 4. September 14. November 1999. Hg. Gabriele Cappellato. Genf, Mailand, Mendrisio: Skira und Accademia di architettura, 1999. 25.

Polaine, Andrew. „The Playfulness of Interactivity“. Proceedings of the 4th International Conference on Design and Emotion 2004. Hg. Aren Kurtgözü. (Middle East Technical University, Ankara und The Design and Innovation Society, 2004). https://zenodo.org/ record/2619748\#.XLxnaaTgqUk (20. April 2019).

Pommer, Richard. „Postscript to a Post-Mortem“. Idea as Model. Hg. Ders. New York: Ausstellungskatalog Institute for Architecture and Urban Studies, 1981. 10-15.

Raulff, Ulrich. „Image oder Das öffentliche Gesicht“. Das Schwinden der Sinne. Hg. Dietmar Kamper und Christoph Wulf. Frankfurt a.M.: Suhrkamp, 1986. 46-58.

Sala, Nicoletta und Gabriele Cappellato. Viaggio matematico nell'arte e nell'architettura. Presentazione di Mario Botta. Milano: FrancoAngeli, 2003.

Saurwein, Emanuele (Hg.). Der düstre Mantel. Über den aus Holz gebauten San Carlino von Lugano von Mario Botta. Mendrisio: Gabriele Capelli Editore, 2001.

Schneider, Bernhard. „Perspektive bezieht sich auf den Betrachter, Axonometrie bezieht sich auf den Gegenstand“. Daidalos 1: Zeichnung als Medium der Abstraktion, September 1981. 81-95.

Styhre, Alexander. „Disciplining Professional Vision in Architectural work. Practices of Seeing and Seeing beyond the Visual“. The Learning Organization 17.5 (2010): 437-454.

Todt, Severin, Christoph Rezk-Salama und Andreas Kolb. „Virtuelle Rekonstruktion und interaktive Exploration der Schlossanlage Dillenburg“. Virtuelle Welten als Basistechnologie für Kunst und Kultur? Eine Bestandsaufnahme. Hg. Manfred Bogen, Roland Kuck und Jens Schröter. Bielefeld: transcript, 2009. 119-138.

Verstegen, Ute. Vom Mehrwert digitaler Simulationen dreidimensionaler Bauten und Objekte in der architekturgeschichtlichen Forschung und Lehre. 16. März 2007, Vortrag auf dem XXIX. Deutschen Kunsthistorikertag Regensburg.

Von Moos, Stanislaus. „Urbanistica virtuale. Due quasi-monumenti di Mario Botta“. Borromini sul lago. Mario Botta: La rappresentazione lignea del San Carlo alle Quattro Fontane a Lugano. Ausst.Kat. Museo Cantonale d’Arte, 4. September - 14. November 1999. Hg. 
Gabriele Cappellato. Genf, Mailand, Mendrisio: Skira und Accademia di architettura, 1999. 33-37.

Weiss, Philipp. Körper in Form: Bildwelten moderner Körperkunst. Bielefeld: transcript, 2010. Wimsatt, William. „The ontology of complex systems: Level of organization, perspectives and causal thickets“. Biology and Society: Reflections of Methodology, zugleich: Canadian Journal of Philosophy. Hg. Mohand Matthen und Robert X. Ware. Supplementary Vol. 20 (1994): 207-274.

Wittgenstein, Ludwig. Bemerkungen über die Philosophie der Psychologie. Bd. II

(Werkausgabe, Bd. 7). Frankfurt a.M.: Suhrkamp, 1984a.

Wittgenstein, Ludwig. Letzte Schriften über die Philosophie der Psychologie. Bd. III (Werkausgabe, Bd. 7). Frankfurt a.M.: Suhrkamp, 1984b.

\section{Abbildungen}

Abb. 1 Peter Eisenman: House X, 1975-1978. Axonometrische Zeichnung des Schemas H. Quelle: Eisenman 1983, 159, Abb. 209.

Abb. 2 Peter Eisenman: House X, 1975-1978. Axonometrisches Modell des Schemas H. Ansicht von Nordost. Quelle: Eisenman 1983, 163, Abb. 211.

Abb. 3 Peter Eisenman: House X, 1975-1978. Axonometrisches Modell des Schemas H. Ansicht von Norden. Quelle: Eisenman 1983, 164, Abb. 213.

Abb. 4 Mario Botta: S. Carlo alle Quattro Fontane, 1999. Quelle: Cappellato 1999, 241.

Abb. 5 Pierre Huyghe: This is not a time for dreaming, 2004. Still vom Kapitel IV: Le Corbusier träumt ein Drahtgittermodellt. Mit freundlicher Genehmigung des Künstlers.

Abb. 6 Florian Dombois: Zugabe, 2014. Detail. Fotografie der Autorin, Juni 2017.

Abb. 7 Florian Dombois: Zugabe, 2014. Positionierung der Pavillons im Aufriss. Zeichnung aus der Phase 1 des Wettbewerbs, 2012. Quelle: Mit freundlicher Genehmigung des Künstlers. 